This item was submitted to Loughborough's Research Repository by the author.

Items in Figshare are protected by copyright, with all rights reserved, unless otherwise indicated.

\title{
On the use of silver nanoparticles for direct micropatterning on polyimide substrates
}

PLEASE CITE THE PUBLISHED VERSION

http://dx.doi.org/10.1109/TNANO.2011.2160092

PUBLISHER

(c) IEEE

VERSION

AM (Accepted Manuscript)

LICENCE

CC BY-NC-ND 4.0

REPOSITORY RECORD

Ng, Jack H.-G., David E.G. Watson, Joachim Sigwarth, Aongus McCarthy, Kevin A. Prior, Duncan P. Hand, Weixing Yu, Robert W. Kay, Changqing Liu, and Marc P.Y. Desmulliez. 2019. "On the Use of Silver Nanoparticles for Direct Micropatterning on Polyimide Substrates". figshare. https://hdl.handle.net/2134/13403. 
This item was submitted to Loughborough's Institutional Repository (https://dspace.lboro.ac.uk/) by the author and is made available under the following Creative Commons Licence conditions.

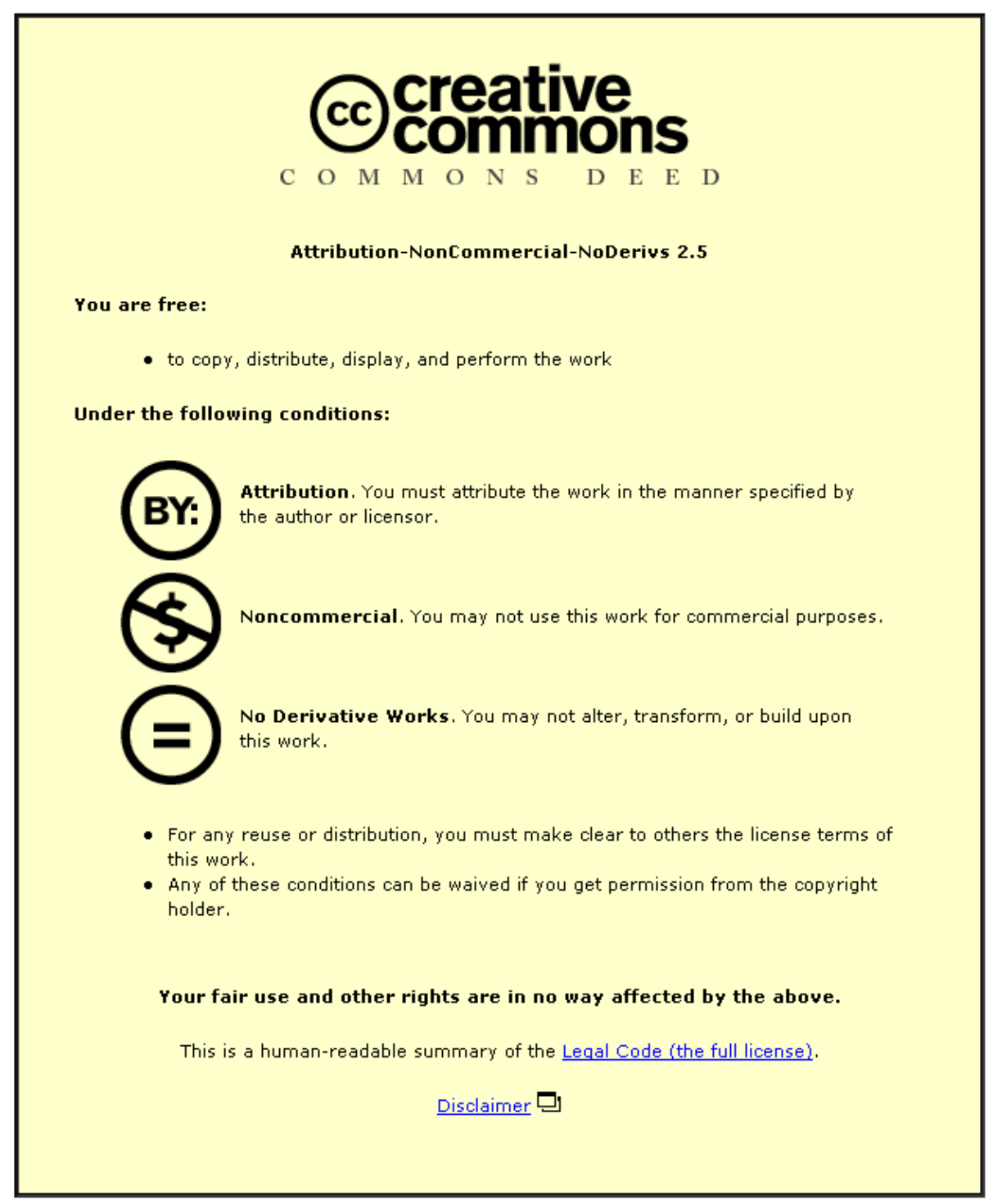

For the full text of this licence, please go to: http://creativecommons.org/licenses/by-nc-nd/2.5/ 


\title{
On the use of silver nanoparticles for direct micropatterning on polyimide substrates
}

\author{
J. H.- G. Ng, D. E. G. Watson, J. Sigwarth, A. McCarthy, K. A. Prior, D. P. Hand, \\ W. Yu, R.W. Kay, C. Liu, Senior Member, IEEE and M. P. Y. Desmulliez, Member, IEEE
}

\begin{abstract}
This article proposes a direct micropatterning process based on the growth of photoreduced silver nanoparticles onto polyimide substrates. The silver nanoparticles are found to have sufficient catalytic efficiency for subsequent electroless plating. Characterization of the process indicates that UV energy dose and heat treatment have to be traded off against photoand thermal degradation of the polymer substrate. Factors affecting the adhesion of the final electroless metal deposit are also discussed.
\end{abstract}

Index Terms - Metallization, photolithography, polyimide, silver nanoparticles, flexible substrate, direct writing, micropatterning, interconnections, packaging, electroless plating.

\section{INTRODUCTION}

$\mathrm{T}$ HE metallization of polyimide substrates has been the subject of intense studies over the last few years as evidenced by the large range of different processes proposed and the number of research groups working in this field worldwide [1-9]. With the increasing industrial relevance of plastic electronics, the race is on for the development of a fast, low cost and reproducible manufacturing process that could replace the current processing techniques such as those used in the printed circuit board industry [10].

Our group recently demonstrated a "bottom-up" technique based on the growth of silver nanoclusters onto an ion-doped polyimide substrate as shown in Fig.1 and

Manuscript received February 2, 2011. This work was supported in part by the UK Engineering Physical Sciences Research Council (EPSRC) project SMART MICROSYSTEMS, referenced FS/01/02/10 and funded by the IeMRC. The work was also financed under the Scottish Funding Council (SFC) funded SRDG Initiative SCIMPS, Scottish Consortium for Integrated MicroPhotonic Systems.

J.H.-G. Ng (phone+ +44 (0)131 451 3783, fax: +44 (0)131 4514155 , email: j.h.ng@hw.ac.uk), M. P. Y. Desmulliez (M.Desmulliez@hw.ac.uk), D. E. Watson (degw1@hw.ac.uk), J. Sigwarth (js208@hw.ac.uk).and R. W. Kay (R.W.Kay@hw.ac.uk) are with the MIcroSystems Engineering Centre at Heriot-Watt University, Edinburgh, EH14 \$AS, Scotland, UK.

D.P. Hand (D.P.Hand@hw.ac.uk), A. McCarthy (A.McCarthy@hw.ac.uk) and K. A. Prior (K.A.Prior@hw.ac.uk) are with the Department of Physics, Heriot-Watt University.

C. Liu (C.Liu@lboro.ac.uk) is with the Wolfson School of Manufacturing and Mechanical Engineering at Loughborough University, Loughborough, LE11 3TU, UK.

W.Yu (yuwx17@hotmail.com) is with the Changchun Institute of Optics, Fine Mechanics and Physics, Chinese Academy of Sciences, No.3888, Southeast Lake Road, Changchun City, Jilin Province, 130033, P.R.China

Copyright (c) 2011 IEEE described in detail in Section II. The growth was further enhanced by a photochemically assistive polymer coating [11]. The resulting deposition process offers several advantages compared to other fabrication routes. Waste materials are drastically reduced compared to traditional subtractive processes. No photoresist and developer materials are necessary, therefore reducing the process running costs. Unlike other mechanical printing techniques, no pre-manufactured silver nanoparticles or inks are required since the silver nanoclusters are produced by in situ photo-reduction. Therefore, the dependency on expensive proprietary products during the fabrication stage is eliminated. Another key advantage is the rigid interface created by these silver nanoparticles mechanically interlocked with the polymer substrate [12], thereby allowing good adhesion of subsequent metallized patterns with the substrate. Sequential laser write or full exposure through a photomask is also possible. Fine pitch lines down to around $15 \mu \mathrm{m}$ have been demonstrated using a continuous-wave laser [11] and around $40 \mu \mathrm{m}$ using UVexposure through a photomask [1]. Finally, the patterning step can be carried out in a dry phase environment and at atmospheric pressure, demonstrating its potential for reel-toreel manufacturing.

Silver nanoparticles are suitable catalysts for the electroless deposition of copper, silver or gold. As well as emerging as a cheaper and easier to prepare alternative to palladium (traditionally used as the catalyst for electroless plating to achieve greater thickness and thus good finishing layer electrical conductivity), processes are being developed that allow fine control over silver nanoparticle size [13],. The micropatterns of active silver surfaces produced from the UV-photomask exposure have a surface morphology that is the result of coalescence or agglomeration of silver nanoparticles. A post-exposure heat treatment step is required to allow the isolated silver particles in the polymer matrix to increase their surface catalytic activity. Higher particle densities and greater surface roughness of the silver seed layer should be produced by higher rates of photoreduction and longer heat treatment times, respectively. These process conditions are desirable for the initiation of subsequent electroless plating. However, prolonged UV irradiation and high temperature treatment can have detrimental effects on the polymer substrate and the process needs optimization to determine the best conditions to generate dense, rough seed metal layers. To date, only preliminary results have been reported $[1,11]$ and in Section III we give a more detailed characterization of the effects of UV energy dose and heat treatment time on the evolution of the silver nanoparticles seed layer and the polyimide substrate. This section also describes the catalytic 
activity of the nanoparticles for initiation of electroless plating along with a discussion on the adhesion of the deposited electroless metal.

\section{EXPERIMENTAL}

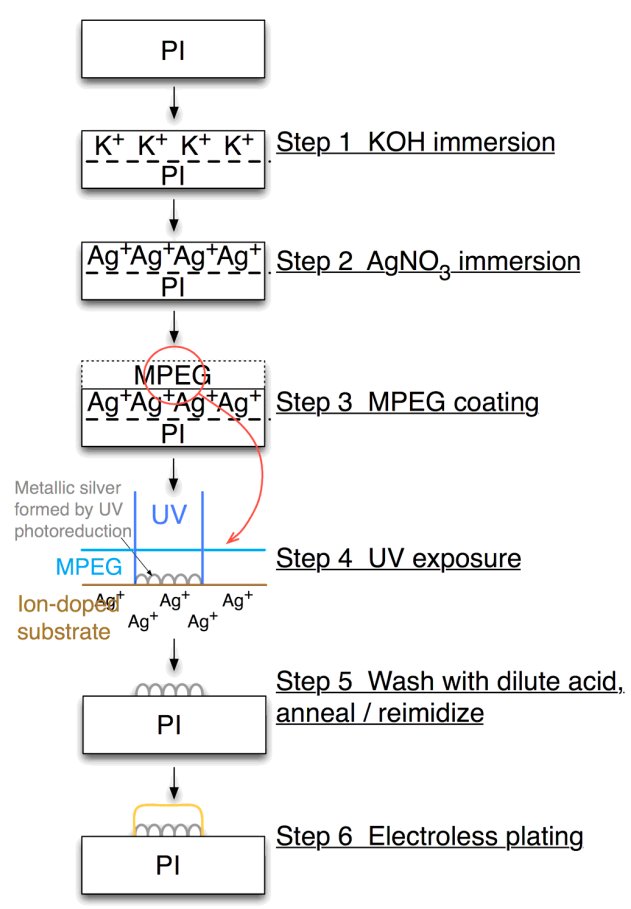

Fig. 1. Process steps of the direct metallization.

\section{Fabrication process}

Fig. 1 shows a schematic process flow of the direct metal micropatterning using UV irradiation on a pre-treated polyimide substrate. The Kapton $200 \mathrm{HN} 50 \mu \mathrm{m}$ thick polyimide films have been obtained from DuPont Inc. and are doped with silver ions through a two-step immersion process. Firstly, the films are immersed in a $1 \mathrm{M} \mathrm{KOH}$ solution at $50^{\circ} \mathrm{C}$ for $5 \mathrm{~min}$ without stirring (step 1). Hydrolysis takes place and a thin surface layer of the polyimide is converted into potassium polyammate. After rinsing with deionized water (DI), the films are then immersed in a $0.1 \mathrm{M} \mathrm{AgNO}_{3}$ solution at room temperature with mild agitation (step 2). The $\mathrm{Ag}^{+}$ions from the solution cause ion-exchange with the $\mathrm{K}^{+}$ions within the modified surface. After rinsing with DI and drying, the silver iondoped polyimide films are spray-coated with a photoactive polymer, methoxy poly(ethylene glycol), (MPEG), using a handheld airbrush (step 3). The MPEG, of $100 \mathrm{~g} . \mathrm{l}^{-1}$ concentration, used absolute ethanol as the solvent, and the solution is heated to completely dissolve the polymer before coating. After coating the MPEG layer, UV-photomask exposure is carried out on the samples with duration ranging from 1 to $8 \mathrm{hrs}$ under a power intensity of approximately 50 $\mathrm{mW} . \mathrm{cm}^{-2}$. The exposure is performed using a broadband mercury arc lamp with roughly collimated light output (step 4). The doped silver ions in the UV exposed areas are photoreduced to zero valence state silver. Subsequently, the MPEG layer is washed off with DI and the samples are then washed in a $1 \mathrm{wt} \% \mathrm{H}_{2} \mathrm{SO}_{4}$ solution. The dilute acid solution allows a second ion-exchange reaction where the $\mathrm{H}^{+}$ions from the solution replace the unreacted $\mathrm{Ag}^{+}$ions in the unexposed areas of the samples, turning the modified surface layer into poly(amic acid). Finally, the patterned samples are annealed at $300^{\circ} \mathrm{C}$ for 1 to $8 \mathrm{hrs}$ in a furnace to induce coalescence of the silver particles (step 5). During annealing, the poly(amic acid) layer also re-imidizes back into the original polyimide molecular structure. Once the desired pattern of the seed silver nanoparticle layer is obtained, electroless plating can proceed (step 6). A commercial autocatalytic electroless silver bath ESM 100 from Polymer Kompositer AB, Sweden has been used in this work for optimized bath performance in terms of bath stability, components monitoring and plating rate.

\section{Characterization}

The silver nanoparticles and aggregates have been compared using a Leo 1530 VP Field Emission Gun Scanning Electron Microscope (FEGSEM). The samples produced from varying UV doses and varying anneal times were also imaged by optical microscopy using an SZX10 Olympus stereomicroscope, and reflectivity measurements were carried out using a continuous-wave $\mathrm{HeNe}$ gas laser at $632.8 \mathrm{~nm}$ (NEC Corporation, Model GLG 5380) and a Newport $1830-\mathrm{C}$ optical power meter at various angles. The etching effect on the polymer substrate was measured by a Veeco Dektak 3 stylus profilometer and a white light phase shifting interferometer (Zygo Viewmeter 5200). FTIR analysis was performed with a Perkin Elmer Spectrum 100.

\section{RESULTS AND DISCUSSION}

\section{Effects of UV exposure dose on the silver particles}

The photo-reduction of silver ions results in the formation of silver atoms within the polymer matrix at the surface of the substrate, which can then immediately nucleate into bigger particles. The applied optimum light dose should therefore high enough so that all silver ions in the exposed areas can be used for photo-reduction. With no annealing and at a relatively low UV energy dose of approximately

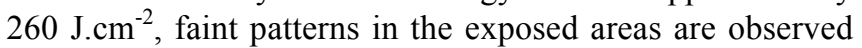

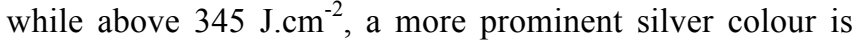
produced. The silver nanoparticles generally display a silvery colour tinged with green or purple as shown in Fig. 2 .

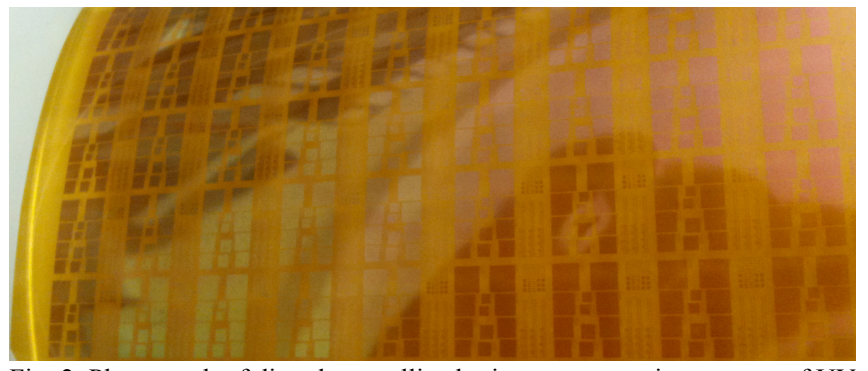

Fig. 2. Photograph of directly metallized micropatterns using a range of UV energy dose irradiations without any heat treatment applied

The FEGSEM images in Fig. 3 show that increasing energy doses increased the density of silver particles formed as expected. Without annealing, the silver particles have a narrow distribution of particle size. However, at UV energy doses above $690 \mathrm{~J} . \mathrm{cm}^{-2}$, the polymer substrate becomes degraded and small holes appear on the substrate amongst the silver nanoparticles. The particle density is found to peak 
at a UV dose of $865 \mathrm{~J} . \mathrm{cm}^{-2}$ although some polymer degradation on the substrate has already occurred by this stage. No clear differences in the particle density can be observed, when the UV dose is increased up to $1210 \mathrm{~J} . \mathrm{cm}^{-2}$ although the polymer substrate becomes increasingly degraded with prominent pores appearing. This plateau of particle density indicates that the source of silver ion source incorporated in the polymer substrate has been depleted. At a dose of $1380 \mathrm{~J} . \mathrm{cm}^{-2}$, the polymer is noticeably degraded with a rough surface containing fewer silver nanoparticles than with doses in the range from 865 to $1210 \mathrm{~J} . \mathrm{cm}^{-2}$.

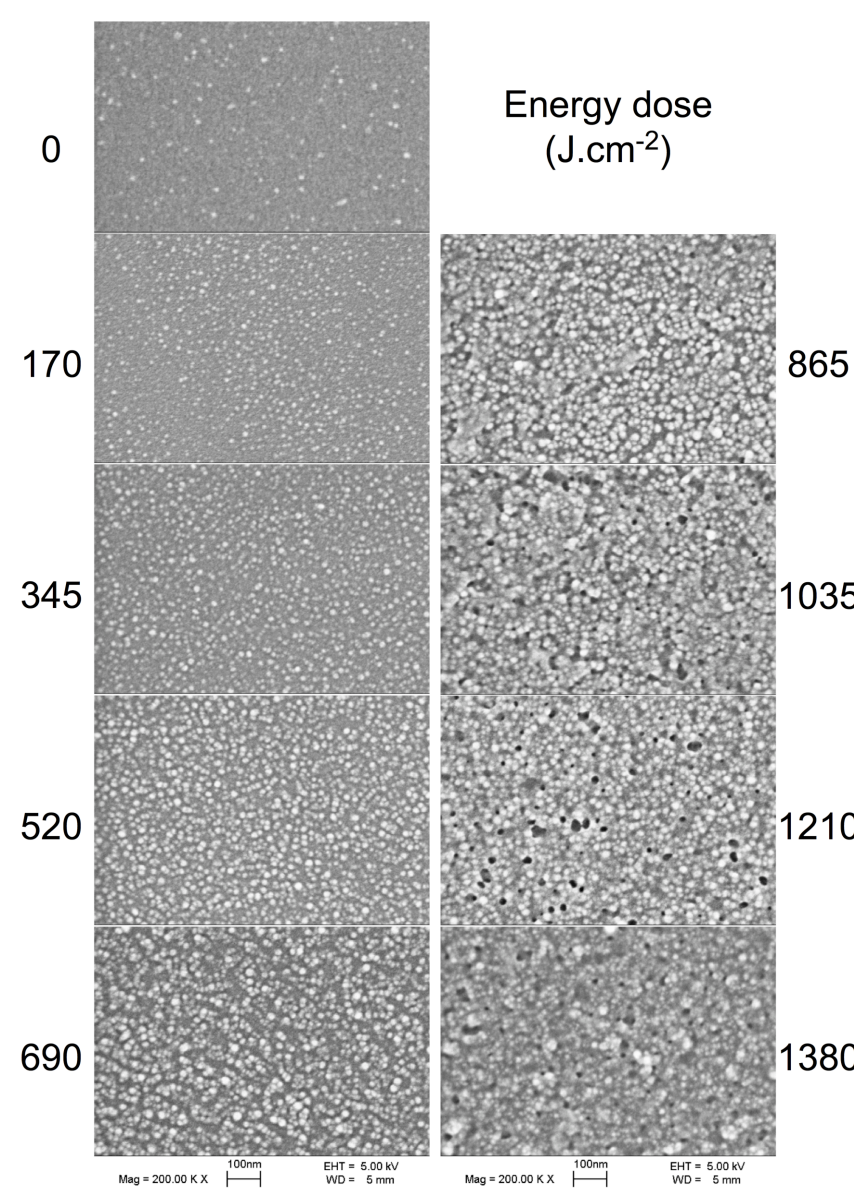

Fig. 3. FEGSEM images of a silver ion-exchanged sample subjected to UV irradiation with different energy dose

\section{Effects of heat treatment}

To produce a silver layer with high surface catalytic activity suitable for subsequent electroless plating, a combination of sufficient dose of light energy and duration of heat treatment is required. The thermal treatment allows the coalescence of the silver particles within the polymer matrix. As a consequence, the silver layer develops a bright silver appearance as shown in Fig. 4(a) compared to Fig. 4(b). Fig. 5 shows a reflectivity measurement of the samples prepared with a fixed UV exposure dose of 690 $\mathrm{J} . \mathrm{cm}^{-2}$ and annealing times varying from 2 to $5 \mathrm{hrs}$. The measurements have been carried out at different incident angles with a laser light source of $632.8 \mathrm{~nm}$ wavelength with maximum power of $0.95 \mathrm{~mW}$. The reflectivity of the samples increases as the annealing time is increased, especially for samples heated for more than $1 \mathrm{hr}$. The reflectivity decreases however beyond $4 \mathrm{hrs}$ of annealing time. With 5 hrs of heat treatment, the exposed areas where silver nanoparticles / polymer composite should be have turned into a brown colour as shown in Fig. 4(b). It can be clearly seen that the polymer regions surrounding the degraded brown patterns also exhibit some changes. This indicates that a thermal effect certainly plays a role in the degradation of the areas that have been exposed to UV radiation. The brown patterns have a roughened surface, which shows an even poorer reflectivity than the sample without heat treatment.

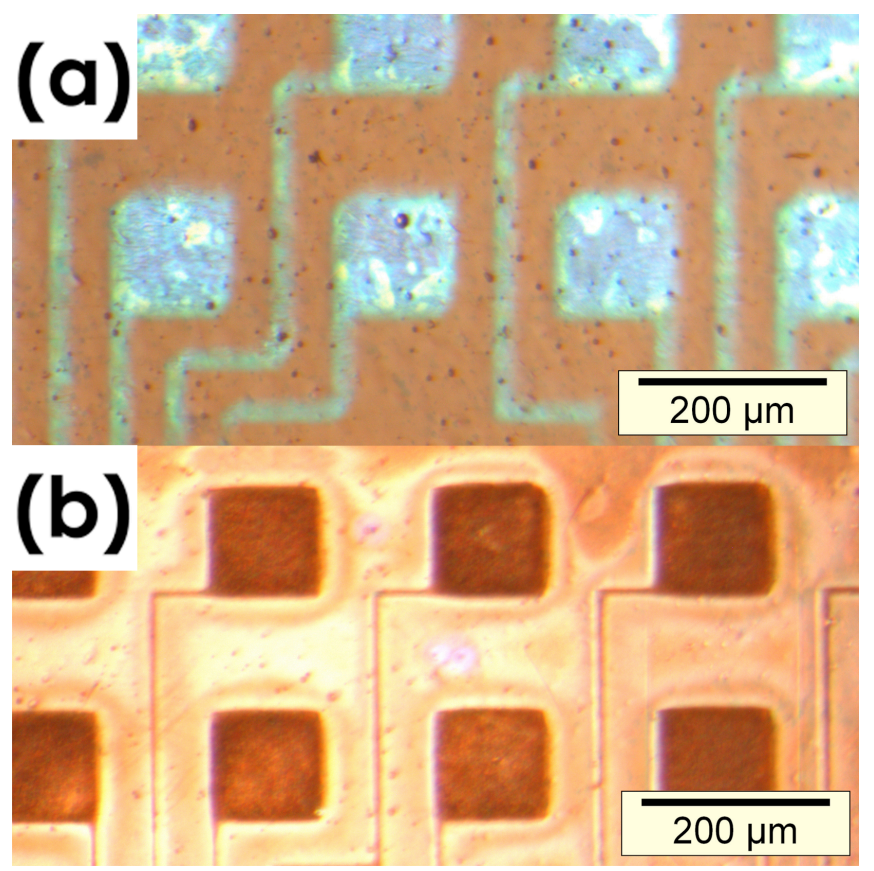

Fig. 4. Optical microscopy images of a sample after photo-reduction with $690 \mathrm{~J} . \mathrm{cm}^{-2}$ and (a) 4 hours heat treatment and (b) 5 hours heat treatment.

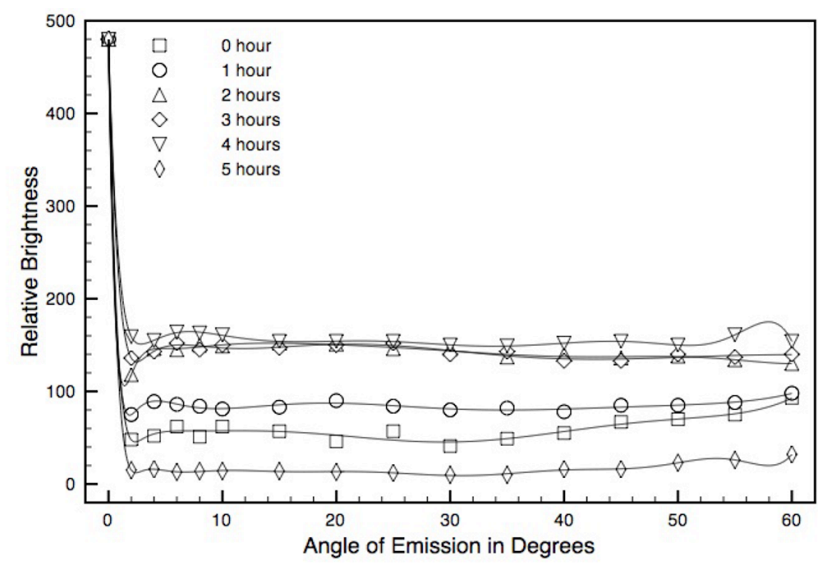

Fig. 5. Reflectivity measurements of a set of samples with fixed UV dose of energy of $690 \mathrm{~J} . \mathrm{cm}^{-2}$ and varying heat treatment times.

Although it is advantageous to choose as short a heat treatment time as possible, the most desirable treatment conditions for these samples are determined by the subsequent electroless plating tests.

The coalescence of the silver particles and degradation of the surface of the substrate occur concomitantly. The evolution of the morphology of the silver nanoparticles can be observed in Fig. 6 from the FEGSEM images of samples with approximately $690 \mathrm{~J} . \mathrm{cm}^{-2} \mathrm{UV}$ exposure energy dose 
and varying annealing times. A virgin polyimide control sample with no chemical pretreatment is also used to isolate any contribution to the substrate of the UV exposure and subsequent annealing. As the duration of the heat treatment increases, the silver treated sample (sample (iii)) displays silver particles with increasing average particle size. When no heat treatment is applied, there is a reasonably regular distribution of silver particles formed by photo-reduction on top of the substrate.

Table 1. Levels of degradation of the surface of the substrate based on FEGSEM images. Samples: (a) unmodified polyimide, (b) unmodified polyimide with UV exposure, (c) $\mathrm{KOH}$ surface modified polyimide, (d) $\mathrm{KOH}$ surface modified polyimide with UV exposure, (e) silver ionexchanged polyimide, and (f) silver ion-exchanged polyimide with UV exposure. A constant UV energy dose of around $690 \mathrm{~J} . \mathrm{cm}^{-2}$ was employed for the exposed samples.

\begin{tabular}{|c|c|c|c|c|c|c|}
\hline $\begin{array}{c}\text { Heat } \\
\text { treatment } \\
\text { time }\end{array}$ & $\begin{array}{c}\text { (a) } \\
\text { PI }\end{array}$ & $\begin{array}{c}\text { (b) } \\
\mathbf{P I}+\mathbf{U V}\end{array}$ & $\begin{array}{c}\text { (c) } \\
\mathbf{K O H} / \mathbf{P I}\end{array}$ & $\begin{array}{c}\text { (d) } \\
\mathbf{K O H} / \mathbf{P I} \\
\mathbf{+} \mathbf{U V}\end{array}$ & $\begin{array}{c}(\mathbf{e}) \\
\mathbf{A g}^{+} / \mathbf{P I}\end{array}$ & $\begin{array}{c}\text { (f) } \\
\mathbf{A g}^{+} / \mathbf{P I}+ \\
\mathbf{U V}\end{array}$ \\
\hline None & no & no & no & textured & no & no \\
\hline $\mathbf{1}$ hour & no & no & no & textured & some & some \\
\hline $\mathbf{2}$ hours & no & no & no & textured & some & some \\
\hline $\mathbf{3}$ hours & no & no & no & textured & moderate & moderate \\
\hline $\mathbf{4}$ hours & no & no & no & textured & moderate & moderate \\
\hline $\mathbf{5}$ hours & no & no & no & textured & severe & severe \\
\hline
\end{tabular}

To determine whether the UV exposure or the annealing step is responsible for the degradation of the substrate, samples annealed for different lengths of time and various treatment conditions and their levels of degradation are shown in Table 1. A control sample with no chemical pretreatment on the polyimide substrate is also used to isolate any contribution to the substrate of the UV exposure and subsequent annealing. These samples, denoted as $(\mathrm{a}-\mathrm{f})$ are as follows: (a) polyimide with no subsequent chemical processing, (b) polyimide with UV exposure and no subsequent chemical processing, (c) $\mathrm{KOH}$ surface modified polyimide with no UV exposure, (d) $\mathrm{KOH}$ surface modified polyimide with UV exposure, (e) silver ion-exchanged polyimide with no UV exposure, (f) silver ion-exchanged polyimide with UV exposure. All samples subjected to UV exposure (samples b, d and f) have been coated with the MPEG polymer. FEGSEM images of these samples can be seen in Fig. 6. As expected, samples $(a-c)$ did not exhibit any significant change in appearance and are therefore not shown here. Columns $(\mathrm{d}-\mathrm{f})$ in Table 1 correspond to columns (i - iii), respectively. A constant UV energy dose of approximately $690 \mathrm{~J} . \mathrm{cm}^{-2}$ was used. All samples with surface modification (samples $\mathrm{c}-\mathrm{f}$ ) have been washed with $1 \mathrm{wt} \% \mathrm{H}_{2} \mathrm{SO}_{4}$ solution prior to anneal. The two polyimide samples with only MPEG coating, no subsequent chemical processing (samples a, b), either with or without UV irradiation both also show no degradation at $5 \mathrm{hrs}$ heat treatment.

Without UV irradiation, the $\mathrm{KOH}$ surface modified sample (sample c) does not exhibit any morphology changes upon heat treatment up to $5 \mathrm{hrs}$. This sample has a similar appearance as the control sample (sample a).

With UV exposure, the same hydrolyzed surface (sample d) shows a "nest-like" texture when no heat treatment is applied, providing thereby a clear indication that the UV irradiation produces the morphology of the surface. When heat treatment is applied, the sample does not exhibit any degradation as observed for samples $d$ and e, but maintains a similar texture throughout the 1 to 5 hours heat treatment.

Without UV exposure, the silver ion-exchanged sample (sample e) starts exhibiting some surface morphological changes at $3 \mathrm{hrs}$ annealing time and after $5 \mathrm{hrs}$, significant degradation of the polymer substrate is observed. Therefore, without UV irradiation the silver ion-exchange and subsequent dilute acid washing must play a role in inducing the thermal degradation of the polymer substrate.

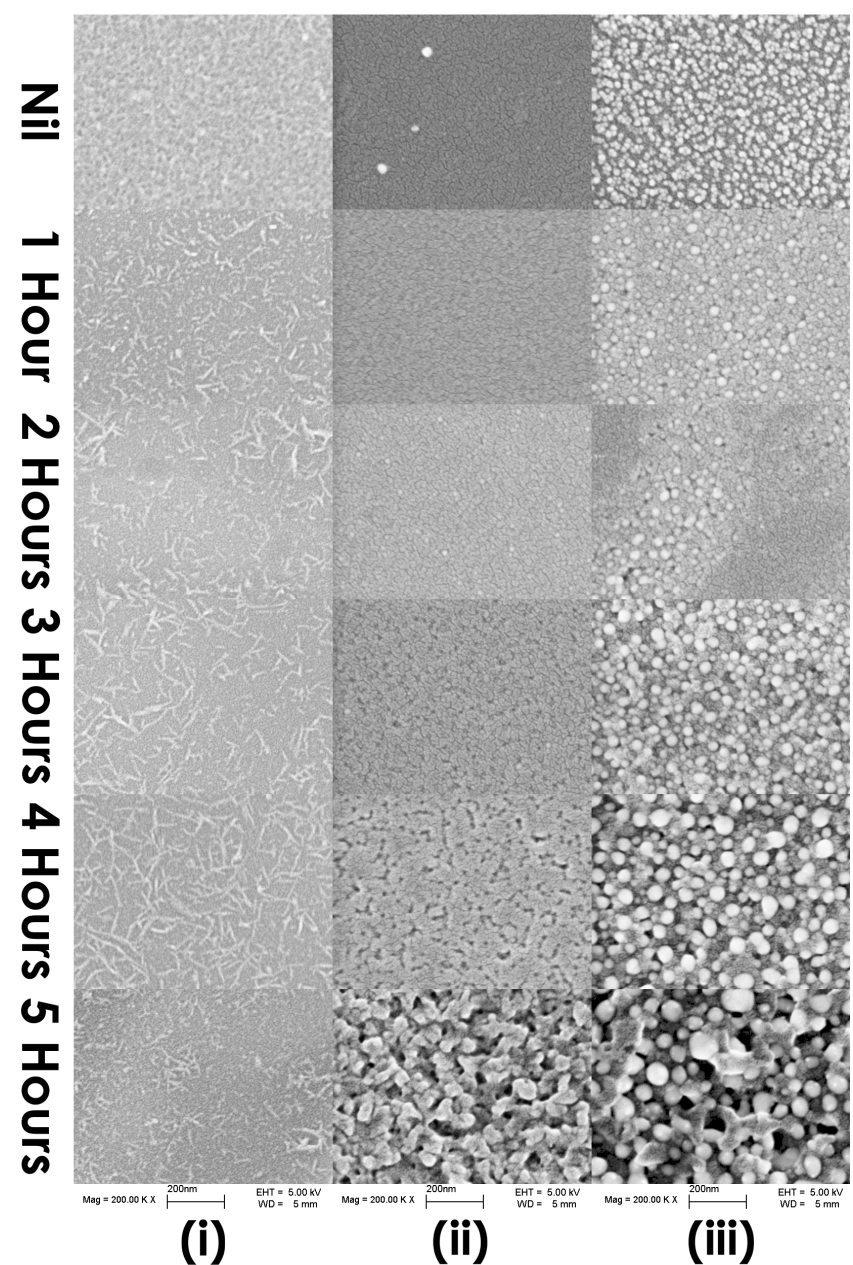

Fig. 6. FEGSEM images of the surface degradation and silver particle coalescence of the samples submitted to different treatment conditions under varying heat treatment times. Samples: (i) $\mathrm{KOH}$ surface modified polyimide with UV exposure, (ii) silver ion-exchanged polyimide, and (iii) silver ion-exchanged polyimide with UV exposure. A constant UV energy dose of approximately $690 \mathrm{~J} . \mathrm{cm}^{-2}$ is employed for the exposed samples.

The silver ion-exchanged sample with UV irradiation (sample f) is the only sample that produces silver nanoparticles. The coalescence of the silver particles and degradation of the surface of the substrate occur concomitantly. The evolution of the morphology of the silver nanoparticles can be observed as the duration of the heat treatment increases. When no heat treatment is applied, there is a reasonably regular distribution of silver particles formed by photo-reduction on top of the substrate without any coalescence taken place. At 1 and $2 \mathrm{hrs}$ heat treatment, the coalescence of the silver nanoparticles is not very pronounced. After 3, 4 and $5 \mathrm{hrs}$ of annealing times, the 
average particle size increases with heat treatment times.

At the same time, the polymer substrate develops leaf-like structures for annealing times above $3 \mathrm{hrs}$. Since no morphology changes are observed for the control sample which underwent the same UV exposure and thermal annealing (sample b), the transformation of the polymer substrate must be due to the chemical treatments. The level of polymer degradation can be seen to be increasing for annealing times between 3-5 hrs. After 5 hrs heat treatment, the degraded polymer shows a leaf-like structure. The silver particles are found on the surface or in the gaps of the fragmented polymer substrate.

The degradation of the surface of the substrate can be explained in terms of oxidative degradation due to UV irradiation and heat treatment in air.

The surface of the substrate is an amorphous polymer containing mobile metal ions, namely potassium polyamate and silver polyamate for the $\mathrm{KOH}$ surface modified samples and silver ion-exchanged samples, respectively. After washing in dilute $\mathrm{H}_{2} \mathrm{SO}_{4}$, hydrogen ions are exchanged with the potassium or silver ions in these samples, forming polyamic acid. These amorphous polymer layers do not exhibit the same good thermal stability of the unmodified polyimide as seen in the samples at $5 \mathrm{hrs}$ anneal without UV irradiation. The extent of degradation is also dependent on the UV energy dose when UV irradiation is applied.

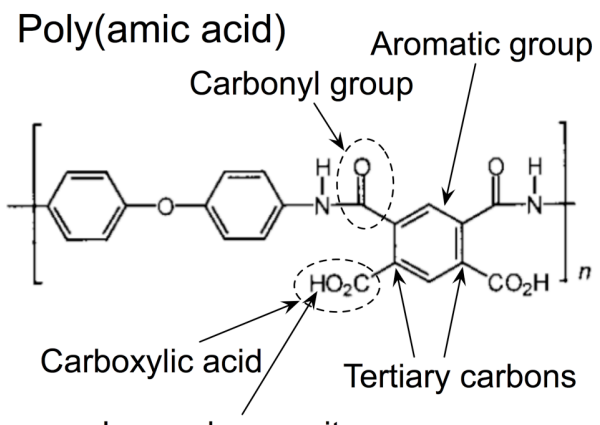

lon exchange site

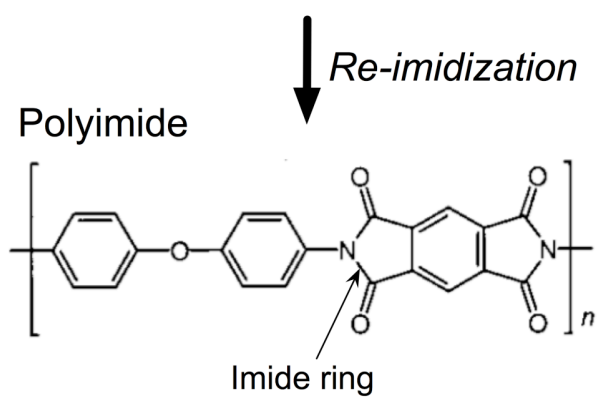

Fig. 7. Structural formulae of poly(amic acid) and polyimide of the type pyromellic dianhydride - oxydianiline (PMDA-ODA).

This suggests that during the heat treatment, oxygen attacks the amorphous layer, which contains tertiary carbon atoms and carboxylic acid, as illustrated by Fig. 7. At elevated temperatures, free radicals form at tertiary carbon atoms are more stable and longer lasting, making them more susceptible to attack by oxygen. Consequently, there is a possibility that the following two reactions can take place together: (1) carbonyl groups can be formed from the oxidation of the tertiary carbon which can then be further oxidized, (2) the neighboring carboxylic acid, composed of a carbonyl group and a hydroxyl group, is already in a high oxidation state and further oxidation removes the carboxyl carbon as carbon dioxide. As a result, some carbon atoms are lost and/or some polymer chains are broken and thereby weakening the poly(amic acid) structure [14].

When continuous UV irradiation is applied, the presence of aromatic groups in the poly(amic acid) molecule can act as a good UV absorbing agent. More free radicals can be formed by the interactions of UV photons with the aromatic groups or the tertiary carbon bonds, which then again react further with oxygen in the atmosphere, increasing the oxidative degradation described above. Therefore, additional damage is observed on the surface modified, UV irradiated samples after heat treatment compared to those not submitted to UV irradiation.

The reaction of poly(amic acid) reforming the $\mathrm{C}-\mathrm{N}-\mathrm{C}$ imide rings upon heat treatment, known as re-imidization, also takes place. Some of the imide rings are indeed reformed after 30 minutes of heat treatment [1]. However, the completeness of this re-imidization process has not been quantified. Whilst re-imidization is taking place, some oxidative degradation effects have already occurred, i.e. some carbon atoms are already removed or the polymer chains are already shortened, especially in the UV irradiated regions of the samples. This could affect the crosslinking of the reformed polyimide molecules and hence the layer of polyimide recovered from the modified material may not possess the same physical properties as those of the unmodified polyimide in the bulk of the substrate.

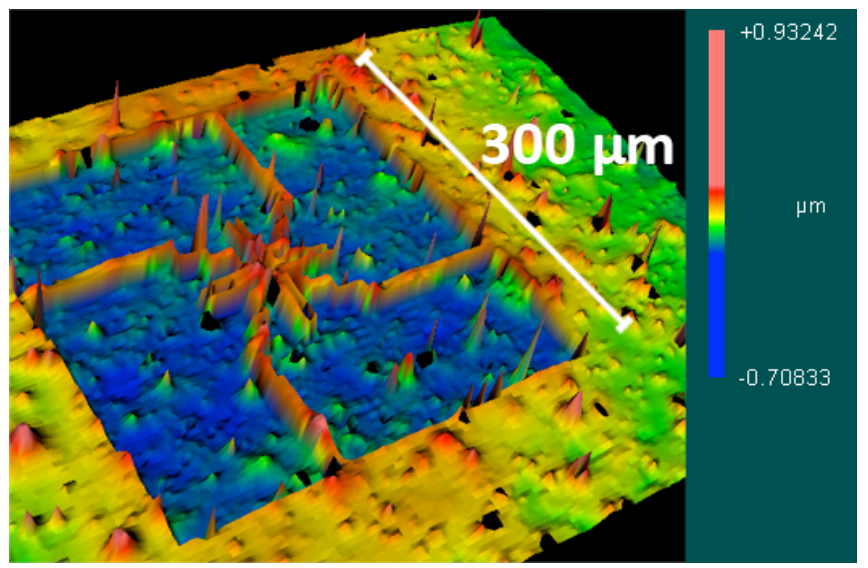

Fig. 8. Height profiles measured by Zygo.

\section{Further degradation effects on the UV irradiated and annealed areas}

The silver patterns on the UV exposed and annealed samples were found to be recessed into the substrate as shown in the Zygo white light interferometer height profile in Fig. 8. This phenomenon may be attributed to polymer deterioration due to the oxidative degradation described in the previous section. The depth of the recession is quite uniform across the whole microstructure exposed to UV light. The Dektak stylus profiler measurements across a UV exposed pattern, shown in Fig. 9, confirms that the recessed areas are up to a few hundred nanometers deep with silver nanoparticles residing at the bottom of the recessed patterns.

As discussed earlier, the oxidative degradation on the substrate is believed to be due to a combination of heat treatment and UV irradiation. To obtain further evidence for this hypothesis, the influence of the annealing time and UV 
energy dose on the size of the recess has been investigated.
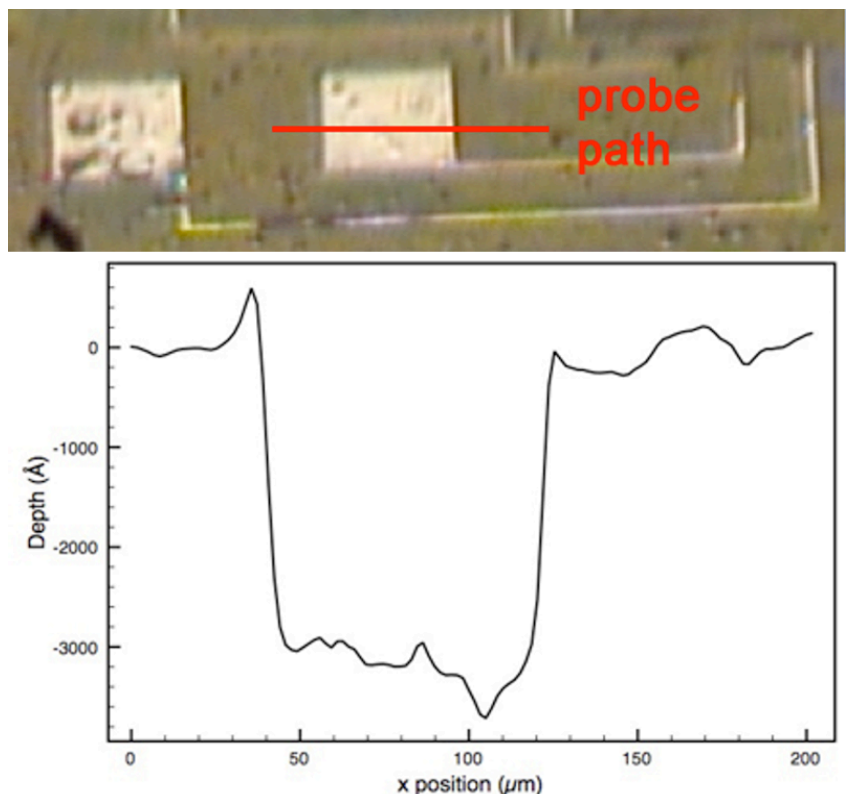

Fig. 9. Dektak stylus profiler measurement across an $80 \times 80 \mu \mathrm{m}^{2}$ contact pad with silver seed layer fabricated by $690 \mathrm{~J} . \mathrm{cm}^{-2} \mathrm{UV}$ dose and 4 hours anneal time.

The graph in Fig. 10 shows that the depth of recess increases with annealing time for a range of different UV exposure times. Clearly the longer a sample is annealed for, the greater the recess depth. However it appears that no further degradation occurs after between approximately 4 to 6 hours, depending on the previous UV energy exposure time, where a plateau is reached. The shorter UV exposure times, i.e. the samples with the lower energy doses before annealing, take longer to reach a plateau than those given a higher UV energy dose. It is also evident from Fig. 10 that the UV exposure plays a part in the degradation, with recess depth from an 8-hour UV exposure being approximately $50 \%$ greater than a 2 -hour exposure. These results clearly indicate that the polymer shrinkage can be attributed to a combination of both photo- and thermal effects.

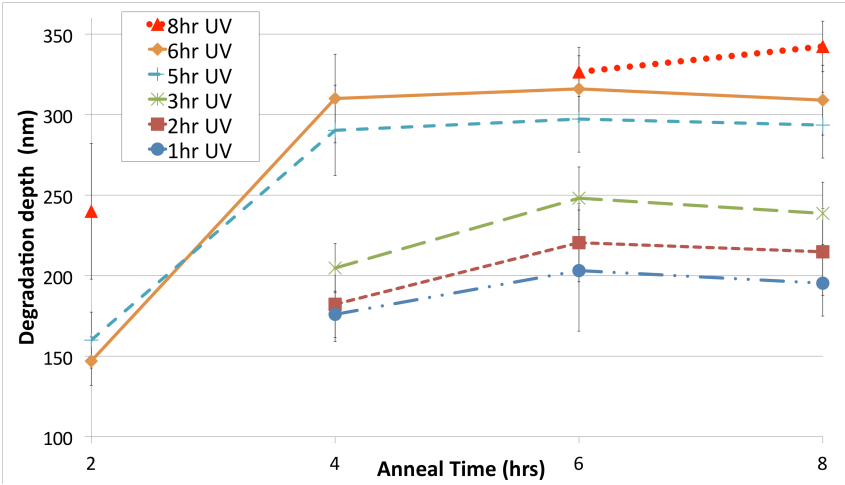

Fig. 10 Effect of annealing time on depth of substrate degradation for selected UV exposure times.

To articulate how these effects combine to produce this phenomenon, we present a tentative hypothesis of the physical mechanisms at molecular level. The longer the UV exposure time, the more silver nanoparticles will coalesce [12]. Also, it has been shown that incompletely imidised polyimide is liable to break down into smaller molecules, mainly in the form of carbon monoxide and carbon dioxide
[14-17]. Furthermore, it has been reported that the presence of silver on a polyamic acid interface can inhibit reimidisation [18]. It is possible that this effect, combined with the silver particles acting as thermal radiators, during both the photochemical and thermal steps of the process, will accelerate the decomposition of the Kapton substrate. Attenuated total reflection Fourier Transform Infra-Red spectroscopy (ATR-FTIR) has been measured on the recessed areas. The spectra (results not shown) were found to be almost identical to pristine polyimide. The degraded polymer top layer is believed to be too thin such that the surrounding unmodified polyimide molecules contribute to the FTIR signals collected.

Since the samples with heat treatment only and without UV irradiation did not have any pattern, whether this recession effect took place on those samples was not investigated and will need to be the study of further research.

\section{Electroless plating}

Electroless plating has the advantage of not requiring an applied voltage since it is a chemically driven process, eliminating the use of electrical contacts and the need to determine the electrical field distribution. This plating method is also useful for the metallization onto nonconducting surfaces or 3-dimensional structures.

An electroless bath solution is composed of specially selected chemical components with determined concentrations. For effective electroless plating, the $\mathrm{pH}$ and the temperature of the bath are also carefully monitored. Bath stability is maintained until electron transfer is initiated. Initiation of electroless plating is usually achieved by the presence of suitable catalysts on the surface of the substrate. The catalysts can serve as metal nucleation sites, adsorbing the target metal ions, reducing molecules from the plating solution onto their surfaces and lowering the activation energy of the plating reactions.

In order to create sufficient numbers of catalytic sites for the initiation of electroless plating, samples with UV irradiation of $690 \mathrm{~J} . \mathrm{cm}^{-2}$ have been processed to ensure that the photo-reduction process used up all the available silver ions for the production of silver nanoparticles. Subsequent heat treatment has increased the catalytic activity of the silver surfaces by allowing the nanoparticles to coalesce into larger particles. Samples with different heat treatment times are compared to test their performances in the electroless bath. The electroless silver bath ESM 100 chosen for this study is autocatalytic. The deposited layer acts as the catalyst for further plating, once the initial catalysts on the substrate are covered by the plated silver.

The key parameters for controlling this bath are the cyanide to silver ion ratio ( $\mathrm{CN}: \mathrm{Ag}), \mathrm{pH}$ and temperature. They can be tuned to suit the catalytic activity of the silver nanoparticles, however any adjustments need to be within the range of operating limits engineered for the various chemical reactions in the bath. Cyanide is a ligand which prohibits the release of the silver ions in the bath and therefore, if the $\mathrm{CN}: \mathrm{Ag}$ ratio is too high, no plating occurs while if it is too low, overplating occurs and the rapidly plated layer detaches itself easily from the substrate. Moreover the substrate areas without any catalysts can nucleate a plated area during overplating. $\mathrm{CN}: \mathrm{Ag}$ ratios of 
0.79-1.1 were tested. The optimum operating temperature of the bath was $67^{\circ} \mathrm{C}$ but temperatures as low as $55^{\circ} \mathrm{C}$ were tested, and it was found that when the temperature is too low the plating will not occur. Similarly, variations of $\mathrm{pH}$ between 9.8 and 10.3 were also tested and the optimum $\mathrm{pH}$ was 10 .

In any case, it is preferable to increase the catalytic activity of the silver nanoparticles rather than adjusting the bath parameters away from their optimum operating conditions. This ensures the best qualities of the deposited layer including hardness and adhesion, whilst minimising the number of parameters to be configured.

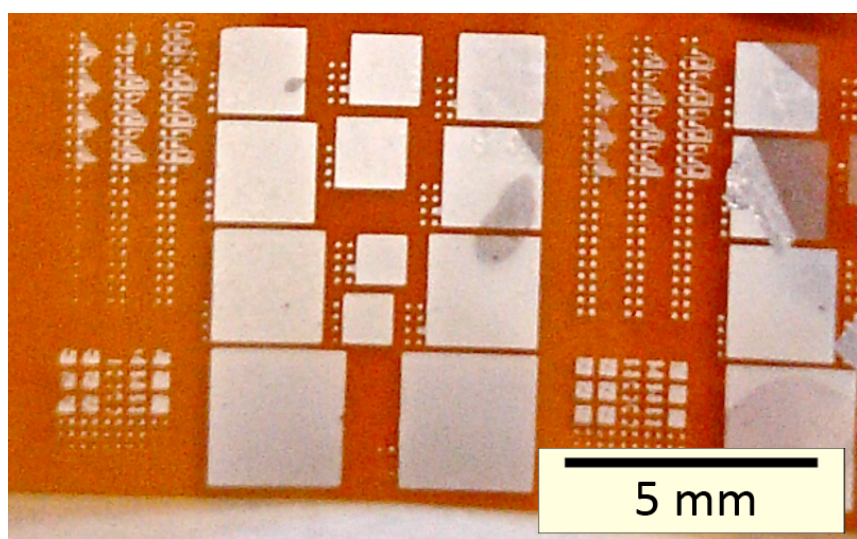

Fig. 12. Optical micrograph of the 4 hours heat treatment sample plated with electroless silver.

To increase the catalytic activity of the silver nanoparticles, heat treatment time has been increased to allow further coalescence. Evidence of the transformation of the silver nanoparticles was described earlier in the reflectivity measurements and the FEGSEM images. The $2-$ 4 hours heat treated samples exhibit the highest reflectivity and have been tested against the electroless bath for their catalytic activity performance.

With no heat treatment, all silver nanoparticles dissolved into the plating solution, suggesting that without any heat treatment, no re-imidization takes place so the modified layer is susceptible to attack by the plating solution.

After 2 hours heat treatment, the plating is not initiated within 10 minutes. For a seed layer with high catalytic activity, the electroless plating should proceed within a minute with the deposited layer visibly observable.

After 3 hours heat treatment, plating is initiated and proceeds selectively onto the regions containing silver nanoparticles. However, the plated layer soon detaches from the substrate into the bath.

The best plating result is found to be with 4 hours heat treatment, with bath parameters of $\mathrm{CN}: \mathrm{Ag}=1.06, \mathrm{pH}=9.9$, and temperature of $67^{\circ} \mathrm{C}$. The deposited electroless silver has a rough white appearance as shown in Fig. 12. A magnified image of the sample under an optical microscope is shown in Fig. 13. The minimum feature size that allowed the deposited electroless silver layer to remain on the sample was about $30 \mu \mathrm{m}$. The tracks with line width between $10-$ $20 \mu \mathrm{m}$ appeared to have been attacked by the plating bath. Not only was no plated layer found on those patterns, but the original silver nanoparticle seed layer had also disappeared. It is believed that with such narrow line width the plating solution could more readily attack the degraded polymer underneath the silver nanoparticle layer, resulting in the detachment of the seed layer. Fig. 13(b) shows that when the sample was immersed in the plating bath for a longer period, the electroless silver layer deposited on the bigger feature patterns also starts to disappear. It can be seen that the detachment was not between the electroless silver layer and the silver nanoparticle seed layer. There is no seed layer like that shown in Fig. 4 left behind. The degradation of the polymer substrate, clearly seen in the FEGSEM image in Fig. 6, causes a severe impediment to the mechanical interlocking between the polymer and the silver nanoparticles, and as a consequence poor adhesion of the deposited metal results.

In addition, it can be also observed from the FEGSEM images that the silver nanoparticles are not closely packed together in any of the samples. In fact, the silver nanoparticles only occupied no more than $50 \%$ of the substrate with areas of polymer gaps in between them. The low density of the silver nanoparticles would provide a weak adhesion strength to any electroless layer plated on top and it also exposes more of the degraded polymer to the attack of the plating solution.

The maximum density of the silver nanoparticles on the substrate produced by the photo-reduction, can only be increased by increasing the loading of silver ions in the substrate. This can be achieved by increasing the degree of $\mathrm{KOH}$ surface modification initially and thus a larger volume of silver ions can be exchanged into the substrate. However, a high degree of surface modification on polyimide using high concentration of $\mathrm{KOH}$ solution has been shown to cause severe degradation to the rigidity of the polyimide film [1]. Therefore a balance needs to be established between sufficient loading of silver ions for effective subsequent electroless plating and degree of $\mathrm{KOH}$ surface modification.
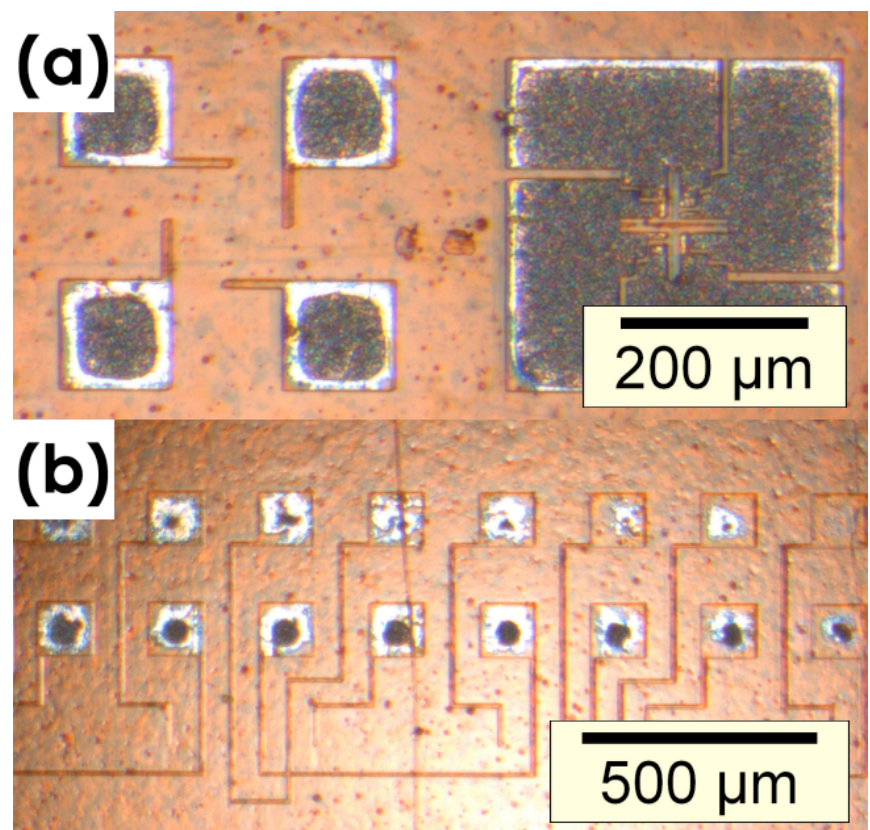

Fig. 12. Magnified optical micrographs of the sample shown in Fig. 11. (a) shows the disappearance of the narrow tracks; (b) shows the electroless silver deposited on the bigger features also disappeared with longer immersion period in the plating bath. 


\section{CONCLUSIONS}

This article has presented the various process parameters affecting the production of a suitable silver seed conductive layer generated on top of a polyimide substrate. The conditions for substrate preparation, photo-reduction, initiation of electroless plating and adhesion of the deposited metal have been reviewed and their competing effects explained.

The applications of various UV energy doses and heat treatment times have been presented in the context of the surface modification of the polyimide substrate. Increasing the UV energy dose increases the amount of silver nanoparticles formed. When a critical UV energy dose is employed, all the silver ions available in the exposed areas of the substrate are used for photo-reduction. At energy dose above $690 \mathrm{~J} . \mathrm{cm}^{-2}$, degradation of the surface modified polymer substrate is observed. UV irradiation was also shown to cause morphology changes to the sample with $\mathrm{KOH}$ surface modification only without incorporation of silver ions. This change remained constant upon increasing heat treatment times. Heat treatment of silver nanoparticles induced two antagonistic effects: particle growth and polymer degradation. Possible causes of the oxidative degradation of the polymer substrate have been suggested.

Suitable catalytic activity of the silver nanoparticles is required for the initiation of electroless plating. Such an activity has been shown to increase with increased heat treatment time.

Adhesion of the electroless metal deposits has been shown to be affected by the degradation of the polymer substrate due to heat treatment and also the insufficient density of the silver nanoparticles as a seed layer.

Increases of both the catalytic activity of the silver nanoparticles by longer heat treatment and of the density of the silver nanoparticles by higher loading of silver ions in the substrate are recommended to optimize the performance of the final electroless plating. However a compromise must be reached between such measures and the degradation of the substrate due to the heating of the modified polymer and/or the high surface modification due to increased $\mathrm{KOH}$ concentration. To reduce the processing times for industrial adaptation, flash exposure lamps [19] or various types of lasers can be employed, and this is currently being pursued by our group.

\section{ACKNOWLEDGMENTS}

J. H.-G. Ng thanks A. Remgård and C. Göransson of Polymer Kompositer AB, Sweden, for the useful discussions and advice on the operation of the ESM electroless bath. The authors would also like to thank Mr J. Bates from Loughborough University for carrying out the FEGSEM microscopy.

\section{REFERENCES}

1. J.H.-G. Ng, M.P.Y. Desmulliez, et al., "A direct-writing approach to the micropatterning of copper onto polyimide", Circuit World 35(2): 3-17, Apr. 2009.

2. J.H.-G. Ng, D.E. Watson, et al, "An additive method for photopatterning of metals on flexible substrates", Proceedings of 36th International MATADOR Conference, Springer, 9-6(389 - 392), July 2010 .
3. M. Tagawa, K. I. Maeda, et al., "Atomic beam-induced fluorination of polyimide and its application to site-selective $\mathrm{Cu}$ metallization." Langmuir 23: 11351-11354, Nov. 2007.

4. Y. Matsumura, Y. Enomoto, et al. (2010), "Fabrication of Copper Damascene Patterns on Polyimide Using Direct Metallization on Trench Templates Generated by Imprint Lithography" Langmuir 26(14): 12448-12454, July 2010.

5. S.C. Park and Y.B. Park, "Effect of Temperature/Humidity Treatment Conditions on Interfacial Adhesion Energy between Inkjet-Printed Ag and Polyimide" Japanese Journal of Applied Physics 48(8), Aug. 2009.

6. O. Berkh, A. Inberg, et al., "Fabrication of flexible microelectrodes using Cold HV sputtering" Journal of Optoelectronics and Advanced Materials 12(5): 1176-1182, May 2010.

7. Y. Liao, B. Cao, et al., "A facile method for preparing highly conductive and reflective surface-silvered polyimide films" Applied Surface Science 255(19): 8207-8212, July 2009.

8. S.H. Yoon, J.H. Lee, et al., "Sintering and Consolidation of Silver Nanoparticles Printed on Polyimide Substrate Films" Macromolecular Research 17(8): 568-574, Aug. 2009.

9. J.H. Kim, Y.J. Park, et al., "Surface modification of polyimide film by coupling reaction for copper metallization" Journal of Industrial and Engineering Chemistry 15(1): 23-30, Jan. 2009.

10. N.-S. Kim and K. N. Han, "Future direction of direct writing" Journal of Applied Physics 108(10): 102801-102806, Nov. 2010.

11. J.H.-G. Ng, M.P.Y. Desmulliez, et al., "UV direct patterning of metal on polyimide", IET Micro \& Nano Letters 3(3: 82-89, Sept. 2008.

12. K. Akamatsu, S. Ikeda, et al., "Site-selective direct silver metallization on surface-modified polyimide layers." Langmuir 19(24): 10366-10371, Nov. 2003.

13. S. Jradi, L. Balan, et al, "Spatially controlled synthesis of silver nanoparticles and nanowires by photosensitized reduction", Nanotechnology, 21(9), 095605, Mar. 2010.

14. R. Srinivasan, R.R. Hall, et al., "Chemical Transformations of the polyimide Kapton brought about by ultraviolet-laser radiation" Journal of Applied Physics 78(8): 4881-4887, Oct. 1995.

15. S.F. Dinetz, E.J. Bird, et al., "A comparative study of the gaseous products generated by thermal and ultra-violet laser pyrolyses of the polyimide PMDA-ODA." Journal of Analytical and Applied Pyrolysis 63(2): 241-249, June 2002.

16. Y.N. Sazanov, A.V. Gribanov, et al., "The role of nitrogen atoms in forming the carbon structure in the carbonization of polymer composites." Fibre Chemistry 40(4): 355-364, July 2008.

17. E.E. Ortelli, F. Geiger, et al., "UV-laser-induced decomposition of Kapton studied by infrared spectroscopy." Macromolecules 33(14): 5090-5097, July 2000.

18. J.T. Young, W.H. Tsai, et al., "Characterization of the interface between pyromellitic dianhydride oxydianiline polyimide and silver using surface-enhanced raman-scattering" Macromolecules 25(2): 887-894, Jan. 1992.

19. D.E. O'Hora, W.F. Bueschel, "Flash exposure system for thin photoresist films", Polymer Engineering and Science, 14(7), 509-512, 1974.

Jack H.-G. Ng is from Hong Kong and has lived in Scotland since childhood. He is currently completing his $\mathrm{PhD}$ and also working as a research associate in the Microsystems Engineering Centre (MISEC) at Heriot-Watt University, Edinburgh, Scotland, UK. He obtained his Bachelor degree in Physics from the University of Edinburgh, Scotland, UK where he developed interests in micro / nano-scale sciences. He then obtained a research Master's degree in Nanomaterials from University of Central Lancashire, England, UK and afterwards joined the MISEC group under the supervision of Professors Marc Desmulliez and Duncan Hand. His research interests include deposition and patterning of metals on nonconducting surfaces, wet chemical surface modification, electroless plating, failure modes and upscaling of microfabrication, laser direct-write applications, flexible electronics, and packaging of medical devices. J.H.-G. $\mathrm{Ng}$ is the corresponding author and can be contacted at: j.h.ng@hw.ac.uk

David E. Watson was born in Aberdeen, Scotland and obtained a BEng in elecrronic \& software engineering from Aberdeen University in 2005. After completing an MSc in Microsystems Engineering at Heriot Watt University, Edinburgh in 2008, he is now studying for his $\mathrm{Ph} . \mathrm{D}$ in microsytems in the MISEC group at Heriot Watt University. His main research interests include flexible electronics and MEMS packaging.

Marc P.Y. Desmulliez (M'08) was born in Lille (France) in 1963 and graduated from the Ecole Superieure d'Electricite of Paris, France, in 1987. He obtained two College Diplomas in 1987 and 1989, in Microwave and 
Modern Optics at University College London (London, UK) and in Theoretical Physics at the University of Cambridge (UK). After spending a couple of years in Dunkerque (France) as a Power Engineer, he obtained his $\mathrm{Ph} . \mathrm{D}$. diploma in Optoelectronics in 1995 from Heriot-Watt University, Edinburgh, Scotland, UK. The major fields of study of Prof. Desmulliez are MEMS, advanced packaging and manufacturing technologies.

$\mathrm{He}$ is currently Research Co-ordinator in the Department of Electrical, Electronic and Computer Engineering at Heriot-Watt University, and has been directing the Microsystems Engineering Centre since 1999. This Centre regroups 6 academic members of staff and over $30 \mathrm{Ph} . \mathrm{D}$. students and Researchers, specialized in all aspects of MEMS manufacturing, packaging and testing. Current research interests include the post-CMOS processing of MEMS and advanced 3D manufacturing technologies.

Prof. Desmulliez is a Fellow of the Institute of Engineering and Technology, a member of the IEEE, a Chartered Engineer and a Chartered Physicist from the UK Institute of Physics.

Joachim Sigwarth is from Lenzkirch, Germany. He worked as an internship at the Microsystems Engineering Centre (MISEC) at Heriot-Watt University, Edinburgh, Scotland, UK in 2009 on novel fabrication of flexible electronics and process development and characterisations. $\mathrm{He}$ is currently completing a MSc degree in Microsystems Engineering at the Albert-Ludwigs-Universität Freiburg, Germany.

Aongus McCarthy is from Ferbane, Co. Offaly, Ireland. He graduated with a BSc from University College Galway in 1989, a Diploma in Electronic Engineering from the Institute of Technology in Carlow, Ireland in 1990 and a BSc in Physical Optoelectronics from Essex University, England in 1991. He received a PhD degree in Physics from Heriot-Watt University, Edinburgh, Scotland, in 2002. His research interests include optical interconnects (free-space and guided-wave), optical and optomechanical design, direct laser-writing techniques and microscope systems. He is a Member of the Optical Society of America and the IEEE Laser and Electro-Optics Society.

Kevin A. Prior gained a degree in Natural Sciences in 1976 from Cambridge University, and was awarded a PhD in 1979 in Physical Chemistry from Cambridge University for studies of single crystal metal surfaces. After working as a Research Assistant at Cambridge University, in 1980 he joined the Molecular Beam Epitaxy (MBE) Group at the Post Office (subsequently British Telecom) Research Labs at Martlesham Heath, Suffolk, UK. From 1980 to 1988, he worked on the growth of GaAs, AlGaAs and InP by MBE and also worked on the thermodynamics of MBE growth. In 1988, he moved to Heriot-Watt University, setting up an MBE facility for the growth of wide bandgap II-VI semiconductors. The MBE group worked initially on the development of II-VI semiconductor lasers, and subsequently on the growth, characterization and processing of novel II-VI materials including $\mathrm{MgS}, \mathrm{MnS}$ and $\mathrm{CrS}$, and have published over 150 papers.

Duncan P. Hand is a Professor of Applied Photonics at Heriot-Wat University; he is also currently Head of Physics. His research interests span a range of applications of high-power lasers, primarily in manufacturing and sensing. His research in manufacturing includes laser micro-joining, laser precision machining, and fibre optic delivery of high power laser light; and is a key component of the SMI, an Innovative Manufacturing Research Centre based at Heriot-Watt, which recently received $£ 7.2 \mathrm{~m}$ funding from the UK Engineering and Physical Sciences Research Council.

Changqing Liu received his BEng from Nanjing University of Science and Technology (1985); MSc from the Chinese Academy of Science (1988) of China, and PhD from Hull University, UK (1998). He has worked as Assistant Professor in the Institute of Metals Research of Academia Sinica and as a Postdoctoral Researcher at the IRC in Materials, Birmingham University. From 2000 he joined the Wolfson School of Mechanical and Manufacturing Engineering of Loughborough University, where he is now Professor of Electronics Manufacture. His current research focuses on novel materials and manufacturing processes to enable multifunctional devices and miniaturisation by elaborating the fundamental issues that are primarily associated with the multimaterial, multifunctional, miniaturisation and reliability aspects of electronics-enabled products. He has published over 150 technical papers, and currently is a senior IEEE member.

Weixing Yu was born in Xi'an city of China in 1975. He obtained his Ph.D degree in 2005 in microelectronics from Nanyang Technological University in Singapore. After two years working as a senior engineer in microelectronic packaging industry and a research scientist in $R \& D$ department in Schott, he moved to Heriot-Watt University, Edinburgh, Scotlant, UK, as a postdoc and worked with Prof.Desmulliez on '3-D Mintegration' project financed by EPSRC. In 2009, he moved back to China and work as a research professor in Changchun Institute of Optics, Mechanics and Physics, Chinese Academy of Sciences. His current research interests include micro/nano optics, microelectric packaging, surpace plasmonics for super-resolution nanolithography and so on. He has published more than 30 journal papers.

Robert W. Kay received a B.eng. honors degree in electrical and electrical engineering in 2001 and a Ph.D. degree on the fabrication of microengineered stencils for flip-chip bonding and wafer level packaging in 2008 from Heriot Watt University, Edinburgh, U.K.

As a result of his $\mathrm{Ph}$.D. research, he co-founded the start-up company MicroStencil, Ltd., that now sells its precision electroformed solder paste stencils to the global electronics market. In 2010 he started as postdoctorate research associate on a project looking at post processing of CMOS devices. 